\title{
Hochwasserereignisse und ihre Widerspiegelung im rezenten Seesediment eines Flussstausees
}

\author{
F.W. Junge, K. Jendryschik, P. Morgenstern, H.-C. Treutler, L. Zerling ${ }^{*}$
}

Keywords: ${ }^{137}$ Caesium, high water level, lake sediment, lamination, river reservoir, trace element, Tschernobyl impact

Zusammenfassung: Sedimentkerne aus dem von der Mulde durchflossenen Bitterfelder Muldestausee (Sachsen-Anhalt, Deutschland) sind ein ausgezeichnetes Beispiel für das hohe Potential von Flussstauseen, die bei Hochwasserereignissen eintretenden Veränderungen in der Schwebstoff- und Sedimentqualität objektiv abzuschätzen. Voraussetzung hierfür bilden gesicherte Daten zur zeitlichen Zuordnung der in den Sedimentkernen auftretenden Ereignis- bzw. Hochwasserlagen.

Konnektierungen mit Durchflussdaten der Mulde seit 1975 geben die Möglichkeit, die in den Sedimentkernen auftretenden Ereignislagen konkreten Hochwässern der Mulde mit einem Durchfluss größer $300 \mathrm{~m}^{3} / \mathrm{s}$ zu zuordnen. Darüberhinaus bilden markante Zeitmarken: (a) das durch den Tschernobyl-Fallout hervorgerufene ${ }^{137}$ Cs-Aktivitätsmaximum, (b) der seit der politischen Wende (1989/90) einsetzende Rückgang in der organischen Belastung und (c) der mit der Schließung des Berg-

"Anschriften der Verfasser: F.W. Junge, K. JENDRYschik, Sächsische Akademie der Wissenschaften zu Leipzig, Arbeitsstelle Schadstoffdynamik in Einzugsgebieten, KarlTauchnitz-Straße 1, 04107 Leipzig (Tel.: 03417115318, Fax: 0341-7115344; E-mail: junge@sawleipzig.de); P. Morgenstern, H.-C. Treutler, UFZ-Umweltforschungszentrum Leipzig-Halle GmbH, Permoserstraße 15, 04303 Leipzig; L. ZerLING, Sächsische Akademie der Wissenschaften zu Leipzig, Kommission für Umweltprobleme, KarlTauchnitz-Straße 1, 04107 Leipzig und Hüttenwesens Industrie im Muldeeinzugsgebiet ebenfalls einhergehende Trend abnehmender Element- und Isotopenkonzentrationen.

Am Beispiel eines im September 2002 aus dem Friedersdorfer Becken des Bitterfelder Muldestausees gewonnenen Kurzkernes werden die Ergebnisse der auf verschiedene Methoden gestützten zeitlichen Konnektierung von Sedimentlagen zu konkreten Hochwasserereignissen vorgestellt. Die im Sedimentkern auftretende jüngste und im Ergebnis des August-2002-Hochwassers abgesetzte Ereignislage erweist sich hinsichtlich Mächtigkeit des Sedimentauftrages und geochemischer Eigenschaften als eine besonders markante Hoch-wasserzeitmarke.

Abstract: Studies of sediment cores originating from a reservoir of the River Mulde near Bitterfeld (Saxony-Anhalt, Germany) confirmed that river reservoirs act as excellent archives of flood events. The records were performed by significant changes of the composition and quality of suspended matter and sediment. To combine the investigated sediment core with a reliable time scale, suitable reference data (e.g. radionuclides, geochemical markers) were included into the study. Subsequently, flow data of the Mulde (from 1975 up to now) were used to assign specific floods $\left(>300 \mathrm{~m}^{3} / \mathrm{s}\right)$ to the event layers detected in the sediment cores. In addition to this, further time markers were inspected towards a more reliable adjustment of the time scale. In detail we made use of the ${ }^{137} \mathrm{Cs}$ activity maximum (caused by Chernobyl fallout), as well as of the drop in organic pollution (following the collapse of East Germany's industrial sector 1989/90), and finally of the decrease in the concentration levels of elements and isotopes (a consequence of the reduced activities of mines and metallurgical plants in the River Mulde catchment). 
Exemplarily we present the results for a short sediment core, which originates from the Friedersdorf basin of the Mulde reservoir (sampled in September 2002). The most recent such layer occurring in this sediment core was caused by the flood in August 2002. This event proved to be an outstanding flood time marker with regard to its thickness and geochemical properties.

\section{Einleitung}

Seesedimente, die in weitgehend geschlossenen und maximal mit dem Grundwasserregime in Verbindung stehenden Hohlformen abgelagert wurden (z.B. Maarseen, Toteisseen u.a.), zählen $\mathrm{zu}$ den besten Geo-Archiven für paläoklimatische und paläoökologische Rekonstruktionen der jüngeren geologischen Vergangenheit bis Gegenwart. In ihnen werden die mittel- bis langfristig wirksamen regionalen und globalen Trends der Umwelt- und Klimaveränderungen widergespiegelt. Abrupte, kurzfristige Veränderungen des hydrologischen Kreislaufes (z.B. Hochwässer) werden in diesen nicht an das oberirdische Abflußregime direkt angeschlossenen Sedimentfallen nur unzureichend und mit geringem Auflösungsgrad dokumentiert. Für eine detaillierte Rekonstruktion dieser Ereignisse sind deshalb kontinuierlich durchflossene natürliche oder anthropogen geschaffene Flussstauräume prädestiniert.

Seit 1975 wird die Mulde, einer der am stärksten schadstoffbelasteten Nebenflüsse der Elbe, durch das Restloch des ehemaligen Braunkohlentagebaues Muldenstein geleitet. Langjährige Untersuchungen zur Hydrodynamik, zum Sedimentationsgeschehen und zur Schwermetallbilanz weisen den dadurch entstandenen Bitterfelder Muldestausee (Fläche: $6,1 \mathrm{~km}^{2}$, Volumen: 118 Mio. m³ Einzugsgebiet: $6170 \mathrm{~km}^{2}$ ) als bedeutsame Sediment- und Schadstoffsenke für das Gebiet der unteren Mulde und Elbe aus (Abb. 1; ZerLING et al. 2001). Da die dem Muldestausee zugelieferten Schwebstoffund Schadstofffrachten in extremer Weise von der Wasserführung der Mulde abhängig sind, kommt der Untersuchung des Zusammenhanges zwischen Hochwasserereignis und Depositionsrate eine zentrale Bedeutung zu. Die Fracht eines einziges Hochwasserereignisses kann diejenige einer mehrmonatigen Niedrigwasserperiode mehrfach übersteigen. Abschätzungen zur Depositionsrate sind aus Differenzmessungen zwischen der dem Flussstausee zugeführten und der den Flussstausee passierenden Sediment-

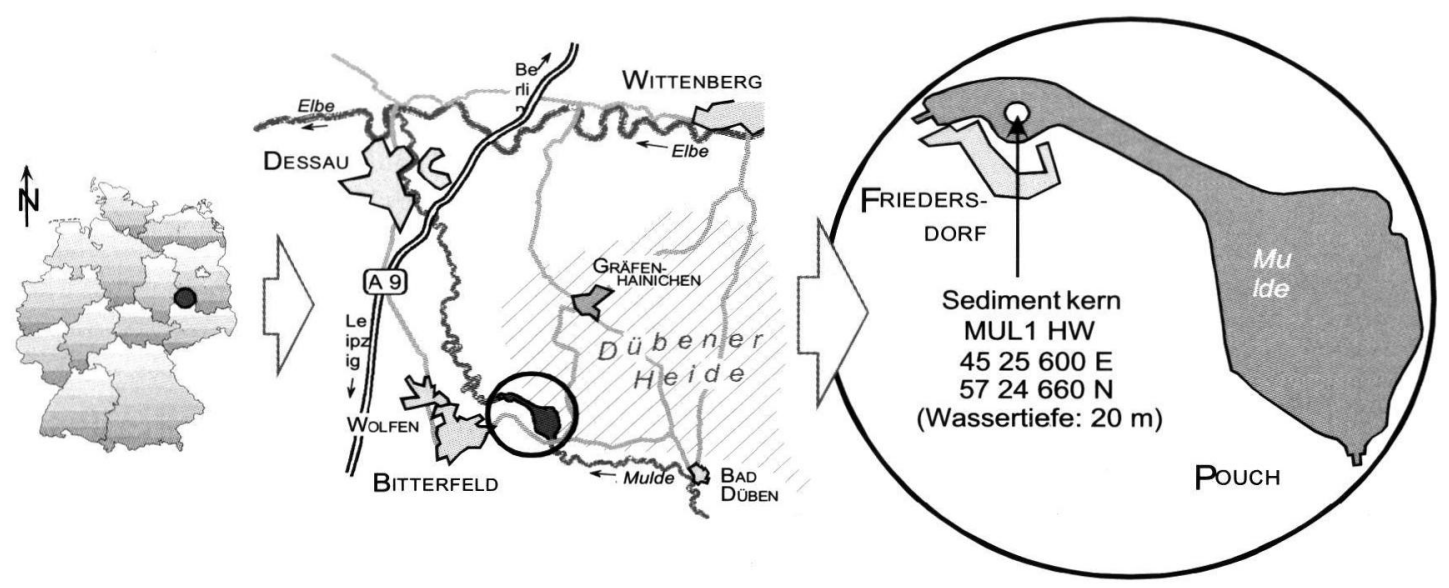

Abb. 1: Geographische Lage des Bitterfelder Muldestausee mit Position des Sedimentkernes MUL1HW. 
fracht möglich und liegen für den Muldestausee als berechnete Monats- bzw. Jahresfrachten seit 1991 vor (JendRYsChik 2003). Für die Verifizierung dieser berechneten Depositionsraten und für eine ereignisspezifische Zuordnung des real im Stausee zur Ablagerung gelangten Schwebstoffes sind Detailuntersuchungen am Seesediment notwendig.

Zu diesem Zweck wurden im April und im September 2002 Sedimentkerne aus dem Friedersdorfer Becken und aus dem Hauptbecken des Muldestausees entnommen. Dabei konnten mit den von einer Schwimmplattform abgeteuften Kernbohrungen die im Muldestausee seit Flutungsbeginn 1975 in den beiden Teilbecken abgesetzten Seesedimente durchteuft und in ihrer Mächtigkeit erfasst werden (Junge et al. 2004). Kurzkerne wurden mittels eines Mondseecorers (Fa. UWITEC, Mondsee/Österreich) insbesondere für die ungestörte Erfassung der jüngsten Seeablagerungen gewonnen. Alle Sedimentkerne wiesen eine deutliche Lamination auf, in denen sich die Hochwasserereignisse der Mulde seit 1975 dokumentieren.

Mit der vorliegenden Arbeit werden die Ergebnisse eines im September 2002 gewonnenen Kurzkernes aus dem Friedersdorfer Becken vorgestellt (Kern MUL1HW: 4525600 Ost, 5724660 Nord; siehe Abb. 1). Den Schwerpunkt der Arbeit bildet dabei die auf verschiedenen Methoden (Gammaspektrometrie, Röntgenfluoreszenzmethode, Hydrologie) beruhende zeitliche Zuordnung der im Sedimentkern dokumentierten Hochwasserlagen.

\section{Methodik}

Probenvorbereitung: Nach Gewinnung des Kernes wurde dieser mittels Folie luftdicht verpackt und bei $4^{\circ} \mathrm{C}$ bis zu seiner Öffnung kühl gelagert. Mit der Kernöffnung im Labor erfolgte die makroskopische Beschreibung, die fotographische Dokumentation und eine Zerteilung des Kernes in Einzelproben entsprechend der Lithologie und den geplanten Untersuchungen. Für geochemische Untersuchungen wurde der Kern MUL1HW in 6 Screeningproben und 25 Detailproben zerlegt, wobei mit letzteren einzelne Sedimentlagen separat erfasst werden konnten (siehe Abb. 2). Die für geochemische Untersuchungen gewonnenen Einzelproben wurden unter Edelgasatmosphäre $(\mathrm{He})$ in Folientüten eingeschweisst und im Gefrierschrank bei Temperaturen kleiner $0^{\circ} \mathrm{C}$ bis zu ihrer weiteren Bearbeitung aufbewahrt.

Gammaspektrometrie: Die gammaspektro- metrischen Untersuchungen erfolgten an den für die Messung in Plastedosen verwahrten separierten Einzelproben des Kernes. Für die Messungen wurden HPGe-Coaxial-Low EnergyDetektoren, n-type mit einem aktiven Volumen von $39 \mathrm{~cm}^{3}$ und einem Berylliumfenster mit $0,5 \mathrm{~mm}$ Dicke eingesetzt. Die Energieauflösung beträgt bei $122 \mathrm{keV}\left({ }^{57} \mathrm{Co}\right) \mathrm{ca} .570 \mathrm{eV}$. An beiden Detektoren wurde die Spektrenauswertung durch die spezielle Software GAMMAW unterstützt. Die Kalibrierung der Detektoren erfolgte mit einem zertifizierten Referenzmaterial der Internationalen Atomenergiebehörde (IAEA). Dabei handelt es sich um Uran-Referenzerz BL5, das mit $\mathrm{SiO}_{2}$ auf einen Urangehalt von 400 $( \pm 2,1) \mu \mathrm{g} / \mathrm{g}$ eingestellt wurde (IAEA-RGU-1), und um Material aus dem Canadian Certified Reference Materials Project (reference britholite ore OKA-2), das mit $\mathrm{SiO}_{2}$ auf einen Thoriumgehalt von $800,2( \pm 15,8) \mu \mathrm{g} / \mathrm{g}$ Thorium (IAEA-RGTh-1) eingestellt wurde. Für die Auswertung der Gammaspektren wurden für die interpretierten Nuklide ${ }^{238} \mathrm{U}$ und ${ }^{137}$ Csdie folgenden Gammaenergien herangezogen: U-238 wird aus dem Tochternuklid bestimmt: ${ }^{234} \mathrm{Th}$ : $63,3 \mathrm{keV}(3,8 \%) ;{ }^{137} \mathrm{Cs} 661,6 \mathrm{keV}(85,1 \%)$. Röntgenfluoreszenzanalyse (RFA). Die Konzentrationsbestimmung für die in dieser Arbeit diskutierten Spurenelemente As, Ba, Cd, Cr, $\mathrm{Cu}, \mathrm{Ni}, \mathrm{Pb}, \mathrm{Sn}, \mathrm{Th}, \mathrm{U}$ und $\mathrm{Zn}$ erfolgte mit der Röntgenfluoreszenzanalyse (RFA) am Gesamt- 
Sedimentkern Bitterfelder Muldestausee (MUL1HW)

18.9.2002, Friedersdorfer Becken (45 25600 E, 5724660 N), Wassertiefe 20 m
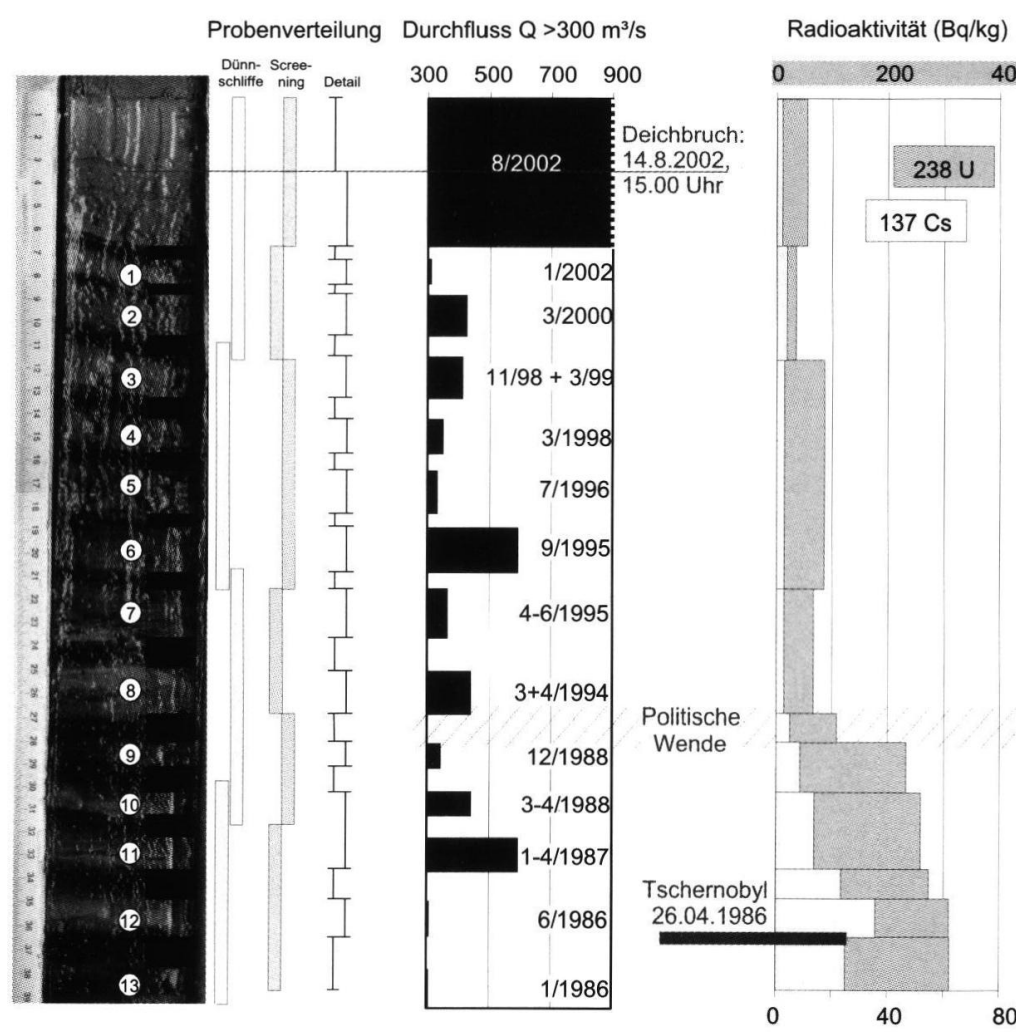

$\mathrm{P}_{2} \mathrm{O}_{5}(\%)$

Metallgehalte $(\mathrm{mg} / \mathrm{kg})$

(P)(C) by EI Zett

Abb. 2: Aufbau des $39 \mathrm{~cm}$ langen Kernes, zeitliche Einordnung und Verteilung ausgewählter Radionuklide $\left({ }^{238} \mathrm{U},{ }^{137} \mathrm{Cs}\right)$, $\mathrm{P}_{2} \mathrm{O}_{5}$ als Indikator der organischen Belastung und Gehalte ausgewählter Elemente und Schwermetalle.

(Die Elementgehalte wurden mittels RFA an der Gesamtfraktion $(<2 \mathrm{~mm})$ bestimmt. In den rechten beiden Kolumnen sind die oberen Skalen gültig für $\mathrm{Zn}$ bzw. $\mathrm{Cu}$; die unteren für As, Pb bzw. Cd, Sn). 
material $(<2 \mathrm{~mm})$. In Vorbereitung zur RFA wurde das vorher mittels einer Achatkugelmühle zerkleinerte Probenmaterial bei $105^{\circ} \mathrm{C}$ getrocknet und anschließend mit $20 \%$ HoechstWachs versetzt, homogenisiert und zu Pellets gepresst. Die analytischen Messungen wurden an einem wellenlängendispersiven Röntgenspektrometer (Siemens SRS 3000) durchgeführt. Dieser Spektrometertyp ist mit einer 3 $\mathrm{kW} \mathrm{Rh}$ - Endfensterröhre (125 $\mu \mathrm{m} \mathrm{Be})$, einem $60 \mathrm{kV}$ Röntgengenerator und Kristallwechsler für acht Analysatorkristalle ausgerüstet. Für die Steuerung des Meßablaufes und die Datenauswertung wurde die Gerätesoftware SPECTRA 3000 benutzt. Die Messungen erfolgten im Vakuum unter Verwendung der $34 \mathrm{~mm}$ Kollimatormaske und unter Einbeziehung der Analysatorkristalle OVO 55, Ge, LIF 200 und LIF 220. Ausführliche Angaben zur verwendeten Methodik und zu den Nachweisgrenzen sind in Junge et al. (2001) enthalten.

\section{Makroskopische Kernbeschreibung}

Der im September 2002 gewonnene ca. 0,40 m lange Kern MUL1HW (4525600 Ost, 5724660 Nord) besteht durchgängig aus laminierten Seesediment mit insgesamt 14 hellen und 13 dunklen Lagen, die eine $\mathrm{cm}$-Wechsellagerung (Lamination) von (a) dunklen, schwärzlich-grauen bis schwarzen organikreichen Lagen und von (b) hellen, gelb bis gelblichgrauen, in unteren Kernbereichen mehr graugefärbten Lagen mit höheren klastischen Anteilen dokumentieren. Nach den makroskopischen Befunden können die hellen Lagen als Ereignislagen in Folge von Hochwässern angesehen werden, die die normale, in den Sommermonaten vor allen biogen gesteuerte und von dunklen Lagen widergespiegelte Seesedimentbildung unterbrechen. Sie sind häufig gradiert und feinlaminiert.

Makroskopisch ist der Kern deutlich zweigeteilt. Der oberste ca. 0,26 m mächtige und insgesamt hell erscheinende Abschnitt besteht aus einer Wechsellagerung von 9 hellen und 8 dunklen Lagen mit scharfen Schichtgrenzen. Der untere ca. 0,14 m mächtige Kernabschnitt macht auf Grund höherer Organikanteile einen dunkleren Eindruck, wobei die Grenzen zwischen den Lagen ( 5 helle, 5 dunkle) mehr verschwommen erscheinen. Die jüngste dunkle Lage des Kernes schließt zeitlich an das Hangende eines im April an derselben Position gewonnenen Sedimentkernes an und spiegelt die überwiegend biogen-bestimmte Sedimentation des Sommerzeitraumes 2002 (April bis 12. August) wider. Darüber ist als jüngste und den Top des Kernes MUL1HW bildende Sedimentschicht eine helle klastische Lage von ca. $55 \mathrm{~mm}$ Mächtigkeit (bei weitgehend unkompaktierter Lagerung) entwickelt. Diese Lage kann dem AugustHochwasser der Mulde zugeordnet werden, das im Zeitraum zwischen dem 13. und 16. August 2002 im Gebiet seinen Höchststand erreichte. Im Sedimentkern erscheint sie hinsichtlich Farbe und Korngröße zweigeteilt. Die unteren $26 \mathrm{~mm}$ sind von gelblichgrauer Farbe und enthalten höhere Anteile gröberen Materials; die oberen $29 \mathrm{~mm}$ sind gelblich gefärbt und weisen höhere Feinstanteile auf. Ursache dieser makroskopischen und lithologischen Zweiteilung der Hochwasserlage könnten die während des August-2002-Hochwassers stattgefundenen Deichbrüche unmittelbar oberhalb des Muldestausees sein (14.8.2002), die zu Schwankungen in der Transportenergie und Schwebstoffführung der Mulde führten.

Erste auf der Basis von Sedimentdünnschliffen ermittelte bisher unpublizierte Korngrößendaten (Methode in Magnus 1998) zeigen, dass das Sediment zu über $90 \%$ aus der $<63$ $\mu \mathrm{m}$-Fraktion (Ton und Schluff) und geringen Anteilen der 63-200 $\mu \mathrm{m}$-Fraktion (Feinsand) zusammengesetzt ist. Nur in den Hochwasserlagen sind gröbere Korngrößenanteile nachweisbar (200-630 $\mu \mathrm{m}$-Fraktion; Mittelsand).

Die mittlere Mächtigkeit aller hellen Lagen 
(ohne Einbeziehung der jüngsten August-2002Hochwasserlage) im Kern MUL1HW beträgt 16,6 mm ( \pm 4,4; Maximum: 25 mm; Abb. 2).

\section{Zeitliche Zuordnung der Hochwasserlagen}

Für die zeitliche Zuordnung der im Kern MUL1HW dokumentierten Hochwasserlagen kamen folgende methodische Ansätze zur Anwendung:

- radiometrische Bestimmung der Konzentrationsverläufe an den Radionukliden ${ }^{137} \mathrm{Cs}$ und ${ }^{238} \mathrm{U}$ im Sedimentkern.

Das durch den Tschernobyl-Fallout (26.4.1986) hervorgerufene eng begrenzte ${ }^{137}$ Cs-Aktivitätsmaximum sollte für den vom Sedimentkern erfassten Zeitraum die Möglichkeit einer punktuellen Zeitmarke liefern. Ebenfalls sollte der mit der politischen Wende 1989/1990 einsetzende abrupte Rückgang des aktiven Uranbergbaus der WISMUT GmbH sich in einem Rückgang der ${ }^{238}$ U-Radionuklid- und Urangesamtgehalte im Sediment widerspiegeln.

- Der 1989/1990 mit der Schließung des Berg- und Hüttenwesens im Muldeeinzugsgebiet ebenfalls einhergehende Trend abnehmender Elementkonzentrationen sollte sich im Seesediment reflektieren (u.a. As, Ba, $\mathrm{Cd}, \mathrm{Cr}, \mathrm{Cu}, \mathrm{Ni}, \mathrm{Pb}, \mathrm{Sn}, \mathrm{Zn})$. Dieser Rückgang sollte unabhängig von den für das Muldeeinzugsgebiet typischen, kontinuierlichen Schwermetallausträgen aus Halden und Grubenanlagen erkennbar sein. Ebenfalls sollte sich die seit der politischen Wende beobachtete geringere organische Belastung der Mulde ebenfalls in der Zusammensetzung des Seesedimentes dokumentieren.

- Auf der Basis der obengenannten "geochemisch verifizierten Zeitmarken" geben die Durchflussdaten der Mulde (Flusspegel Bad Düben; jährlich publiziert in Deutsches
Gewässerkundliches Jahrbuch, LFU Sachsen-Anhalt) die Möglichkeit, die in den Kernen auftretenden Ereignislagen konkreten Hochwässern der Mulde zu zuordnen.

\subsection{Zeitmarken mittels Radiometrie}

Um eine erste Übersicht über die Tiefenverteilung der natürlichen Radionuklide aus der ${ }^{238} \mathrm{U}$-Zerfallsreihe und des anthropogenen ${ }^{137} \mathrm{Cs}$ zu gewinnen, wurden in einer ersten Messserie die 6 Screening-Proben des Kernes MUL1HW untersucht (Abb. 2). Dabei zeigte sich im Teufenbereich zwischen 0,31-0,38 m ein deutliches Aktivitätsmaximum in der ${ }^{238} \mathrm{U}$ $(151 \mathrm{~Bq} / \mathrm{kg})$ und ${ }^{137} \mathrm{Cs}-$ Konzentration (12,3 $\mathrm{Bq} / \mathrm{kg})$. Um aus der ${ }^{137} \mathrm{Cs}$-Tiefenverteilung die Zeitmarke 1986 (Tschernobyl-Reaktorunfall vom 25./26.04.1986) genauer setzen $\mathrm{zu}$ können, wurde dieser Teufenbereich durch die vorhandenen Detailproben ergänzt. Dadurch konnte ein deutliches ${ }^{238} \mathrm{U}$-Konzentrationsmaximum $(309-311 \mathrm{~Bq} / \mathrm{kg}$ im Teufenbereich $0,34-0,38 \mathrm{~m})$ und ein noch ausgeprägteres ${ }^{137} \mathrm{Cs}-$ Konzentrationsmaximum $(35,2 \mathrm{~Bq} / \mathrm{kg})$ gefunden werden.

Im Kern MUL1HW kann auf Grund der ${ }^{137} \mathrm{Cs}$-Verteilung das Sedimentalter von 16 Jahren (Tschernobyl) auf den Teufenbereich von 0,34-0,35 $\mathrm{m}$ eingegrenzt werden. Das ${ }^{137}$ Cs-Konzentrationsmaximum liegt innerhalb einer Hochwasserlage (Ereignislage Nr. 12, Abb. 2), wobei die darunterliegende organikreiche dunkle Lage ebenfalls noch eine erhöhte Aktivitätskonzentration $(24,6 \mathrm{~Bq} / \mathrm{kg})$ aufweist. Der Tschernobyl-Reaktorunfall fand im Frühsommer 1986, in der Nacht vom 25./ 26. April 1986 statt. Hochwasserereignisse mit Maximaldurchflüssen um $300 \mathrm{~m}^{3} / \mathrm{s}$ wurden um den Zeitpunkt des Reaktorunfalls herum am 21.01.1986 (Winterhochwasser) und am 31.5./1.6.1986 (Sommerhochwasser) beobachtet. Aus dieser Konstellation ergibt sich die fol- 
gende Zuordnung der Ereignislagen: Während des Winterhochwassers vom 21.01.1986 kam es zur Sedimentation der untersten, mit dem Kern MUL1HW erfassten Ereignislage (Ereignislage Nr. 13, Abb. 2). Die beginnende organische Sedimentation im Frühsommer 1986 (Zeitraum Februar bis Ende Mai 1986) wird durch die sich darüber befindliche organikreiche, dunkle Lage reflektiert. Innerhalb dieses Zeitraumes ereignete sich der Tschernobyl-Reaktorunfall mit der Folge des Eintrages von ${ }^{137} \mathrm{Cs}$ in das Seesediment. Im Ergebnis des darauffolgenden Sommerhochwassers vom 31.5./1.6.1986 kam es zu einer verstärkten Abschwemmung von ${ }^{137}$ Cs-kontaminiertem Bodenmaterial und damit zu dem beobachteten ${ }^{137}$ Cs-Aktivitätsmaximum innerhalb dieser im Muldestausee abgesetzten Hochwasserlage (Ereignislage Nr. 12, Abb. 2). Seit dieser Zeit führten die jüngeren Muldehochwässer zu einem weiteren, aber abklingenden ${ }^{137}$ Cs-Eintrag in das Sediment des Muldestausees. Die durch den Reaktorunfall von Tschernobyl verursachte Cs-Kontamination, die in einigen Gebieten Deutschlands Belastungen bis $61000 \mathrm{~Bq} / \mathrm{m}^{2}$ erreichte (EINSELE \& Pfeffer 1990), betraf im wesentlichen die obersten organischen Bodenschichten sowie die humosen Mineralbodenhorizonte. Eine Wasserlöslichkeit von Radiocaesium und damit eine einhergehende Verlagerung ist auszuschließen (BöHm \& Pfeffer 1990). Verursacht durch die enge Bindung des Radiocaesiums an die organische Substanz ist allerdings im Zuge von Abspülungsprozessen und dem damit verbundenen Austrag von Bodenfeinmaterial eine Überführung von ${ }^{137} \mathrm{Cs}$ in die Schwebstofffraktion gegeben. Diese Verlagerungsprozesse fanden entsprechend der ${ }^{137} \mathrm{Cs}$-Verteilung im Kern MUL1HW bis zu Beginn der neunziger Jahre statt und können danach dann nicht mehr nachgewiesen werden (ab Ereignislage Nr. 8, Abb. 2). Mit dem Jahrhunderthochwasser im August 2002 war ebenfalls keine erneute ${ }^{137}$ Cs-Mobilisierung verbunden.
Das ${ }^{238}$ U-Radionuklid (Abb. 2) und die Gesamturangehalte zeigen innerhalb des Kernes MUL1HW einen vom Liegenden zum Hangenden abnehmenden Konzentrationsverlauf. Dabei ist im Teufenbereich von 0,24-0,28 m eine sprunghafte Abnahme der ${ }^{238}$ U-Aktivität auf das Niveau der mittleren natürlichen Konzentration zu beobachten. Dieser obere Kernbereich, der gegenüber dem unteren Kernbereich eine um das 6-fache geringere 238U-Aktivität und eine Halbierung des Gesamturangehaltes (20-41 ppm gegenüber 60-78 ppm U) im Seesediment des Muldestausees aufweist, kennzeichnet den Zeitabschnitt der neunziger Jahre und jünger ("Postwendezeit"). In ihm zeigt sich der abrupte Niedergang des Uranbergbaus und der Uranaufbereitung (Zwickauer Mulde; BeUge AT AL. 1994) sowie der Verhüttungsindustrie im Freiberger Bergbaurevier. Die "Vorwende- bzw. DDR-Zeit" (vor 1989/90) ist durch maximale Urangehalts- und Uranisotopenkonzentrationen gekennzeichnet. In den jüngeren Kernabschnitten ist im Zuge von Hochwasserereignissen eine schwache Mobilisierung von ${ }^{238} \mathrm{U}$ nachweisbar (Hochwässer 1995 und 2002).

\subsection{Zeitmarken aus}

\section{elementgeochemischen Untersuchungen}

Gestützt auf die radiometrischen Ergebnisse, erbrachte die Analyse der Screeningproben des Kernes MUL1HW (Abb. 2) auf ihren Spurenelementgehalt und ihren Glühverlust (Organikäquivalent) eine Aufteilung des Kernes in zwei geochemisch voneinander unterscheidbare Sedimentationszeiträume: einen Kernabschnitt, der die „Vorwendezeit bzw. DDR-Zeit“ (1989 und älter; Teufenbereich: größer 0,26 m); und einen Kernabschnitt, der die „Postwendezeit“ (90iger Jahre; Teufenbereich 0 bis $0,26 \mathrm{~m}$ ) widerspiegelt. Sie unterscheiden sich deutlich in ihren Elementgehalten (Gesamtkorngrößenfraktion), wobei das während der DDR-Zeit im Muldes- 
tausee zur Ablagerung gelangte Sediment in der Regel höhere Konzentrationen insbesondere an organischen Elementindikatoren und Schwermetallen aufweist (Tabelle 1).

Im Vergleich zum „Postwendezeitraum “ spiegelt sich der höhere organische Eintrag der Mulde in das während des „Vorwende-Zeitabschnittes" (1989 und älter) abgelagerte Seesediment in deutlich erhöhten Gehalten an Gesamtphosphor (Mittelwert aller untersuchten Kerne: ca. $2,1 \%$ gegenüber ca. 1,1\% des „Postwendezeitraumes"), an Schwefel (im Mittel ca. 1,1 \% gegenüber ca. 0,6\%) und im höheren Glühverlust (Kern MUL1HW: ca. 19 \% gegenüber ca. $15 \%$ in der $<20 \mu \mathrm{m}$-Sedimentfraktion) wider. Umgekehrt werden die geringeren klastischen Anteile innerhalb dieses Kernabschnittes durch niedrigere $\mathrm{SiO}_{2}$ - (im Mittel ca. $45 \%$ gegenüber ca. $53 \%$ ), $\mathrm{Al}_{2} \mathrm{O}_{3^{-}}$(im Mittel ca. $13 \%$ gegenüber ca. $14 \%$; Tonmineralanteil) und Zr-Gehalte (im Mittel ca. 200 ppm gegenüber ca. 120 ppm; Schwermineralanteil) ausgewiesen.

Die zu DDR-Zeiten aus dem aktiven Berg- und Hüttenwesens stammende Freisetzung von Schwermetallen und anderer erzgebundener Elemente wird in dem hohen Konzentrationsniveau der Elemente $\mathrm{As}, \mathrm{Pb}, \mathrm{Sn}, \mathrm{Zn}, \mathrm{Cd}, \mathrm{Cr}$, $\mathrm{Ni}, \mathrm{Cu}$ (siehe Tabelle 1), sowie U (im Mittel ca. $74 \mathrm{ppm}$ gegenüber $30 \mathrm{ppm}$ ), Ba (im Mittel ca. 1372 ppm gegenüber ca. $939 \mathrm{ppm}$ ) und $\mathrm{Sr}$ (ca. 158 ppm gegenüber $117 \mathrm{ppm}$ ) deutlich.

Die Gehalte an den Elementen $\mathrm{Zn}, \mathrm{Cd}, \mathrm{Pb}$, $\mathrm{Cu}$, As und $\mathrm{Sn}$ dokumentieren innerhalb des Kernes vom Liegenden zum Hangenden einen kontinuierlichen Trend abnehmender Konzentrationen. Dabei zeigt sich, vor allem bei den Elementen $\mathrm{Cu}, \mathrm{Cd}$ und $\mathrm{Zn}$ sichtbar, ab einem Teufenbereich von $0,26 \mathrm{~m}$ (ab Ereignislage $\mathrm{Nr}$. 8; Abb. 2) eine sprunghafte Gehaltsabnahme. Damit wird nach elementgeochemischen Befunden die Zeitmarke der "politischen Wende“ (1989/90) fixiert, die mit der Schließung desBerg- und Hüttenwesens im Einzugsgebiet von Zwickauer und Freiberger Mulde einen abrup- ten Rückgang in den Einträgen der genannten Elemente verursachte.

Die Untersuchungsergebnisse der Screeningproben zeigen, dass mit den im Kern MUL1HW dokumentierten Hochwasserereignissen (Ereignislagen 1 bis 13; Abb. 2) keine maßgebliche Erhöhung in den Elementkonzentrationen (Schwermetalle und Erzelemente) im Seesediment verbunden ist. Eine Ausnahme bildet das August-2002-Hochwasser, das an den Bohrpunkten im Muldestausee einen 5-fach höheren Sedimentauftrag auf die Seebodenoberfläche des Muldestausees erbrachte. Dabei sind mit dem Sedimentauftrag deutliche Konzentrationserhöhungen bei den Elementen $\mathrm{Sn}$, As und $\mathrm{Pb}$ im Oberflächensediment nachweisbar, die offenbar während des Hochwassers abgeschwemmtem Haldenmaterial (Freiberger Mulde) entstammen. Hingegen fügen sich die Gehalte der Elemente $\mathrm{Zn}, \mathrm{Cd}, \mathrm{Cr}, \mathrm{Ni}$, Cu (Tabelle 1) und $\mathrm{U}$ des Hochwassersedimentes in den seit den neunziger Jahren allgemein beobachteten Trend einer Verbesserung der Sedimentqualität ein.

\subsection{Hochwasserereignisse und ihre Widerspiegelung im Seesediment} abgeleitet aus Durchflussdaten der Mulde

Für eine verifizierbare Zuordnung der Hochwasserereignisse aus den Durchflussdaten der Mulde zu konkreten Ereignislagen im Seesediment sind die folgenden Überlegungen notwendig: Das Seesediment des Bitterfelder Muldestausees besteht aus einer makroskopisch sichtbaren Wechsellagerung heller Ereignislagen (Hochwasserlagen) und dunkler organikreicher Lagen. Nach den bisherigen Erfahrungen über das Schwebstoffangebot der Mulde bei erhöhten Durchflüssen führen Hochwässer mit einem mittleren Tagesdurchflusswert größer $300 \mathrm{~m}^{3} / \mathrm{s}$ (dies entspricht in etwa dem Fünffachen des mittleren Durchflusses der Mulde am ca. $15 \mathrm{Ki}$ - 
Tab. 1: Mittlere Elementgehalte im Sediment des Bitterfelder Muldestausees in Abhängigkeit vom Depositionszeitraum.

\begin{tabular}{|c|c|c|c|c|c|c|c|}
\hline Zeitraum & $n$ & $\begin{array}{c}\text { As } \\
\mathrm{mg} / \mathrm{kg}\end{array}$ & $\begin{array}{c}\mathbf{P b} \\
\mathrm{mg} / \mathrm{kg}\end{array}$ & $\begin{array}{c}\mathrm{Sn} \\
\mathrm{mg} / \mathrm{kg}\end{array}$ & $\begin{array}{c}\mathrm{Zn} \\
\mathrm{mg} / \mathrm{kg}\end{array}$ & $\begin{array}{c}\mathbf{C d} \\
\mathrm{mg} / \mathrm{kg}\end{array}$ & $\begin{array}{c}\mathrm{Cu} \\
\mathrm{mg} / \mathrm{kg}\end{array}$ \\
\hline $\begin{array}{l}\text { August } 2002 \text { - } \\
\text { Hochwasserlage }\end{array}$ & 2 & $\begin{array}{c}251 \\
( \pm 96) \\
(183 \ldots 319)\end{array}$ & $\begin{array}{c}\mathbf{5 3 7} \\
( \pm 192) \\
(401 \ldots 673)\end{array}$ & $\begin{array}{c}64 \\
( \pm 6) \\
(59 \ldots 68)\end{array}$ & $\begin{array}{c}1225 \\
( \pm 59) \\
(1183 \ldots 1266)\end{array}$ & $\begin{array}{c}21 \\
( \pm 1) \\
(20 \ldots 22)\end{array}$ & $\begin{array}{c}160 \\
( \pm 20) \\
(146 \ldots 174)\end{array}$ \\
\hline $\begin{array}{l}\text { "Postwende- } \\
\text { zeitraum" (1989/90 } \\
\text { bis August 2002) }\end{array}$ & 13 & $\begin{array}{c}171 \\
( \pm 23) \\
(135 \ldots 216)\end{array}$ & $\begin{array}{c}294 \\
( \pm 16) \\
(262 \ldots 330)\end{array}$ & $\begin{array}{c}44 \\
( \pm 9) \\
(36 \ldots 72)\end{array}$ & $\begin{array}{c}1676 \\
( \pm 127) \\
(1447 \ldots 1885) \\
\end{array}$ & $\begin{array}{c}34 \\
( \pm 7) \\
(27 \ldots 54)\end{array}$ & $\begin{array}{c}170 \\
( \pm 22) \\
(148 \ldots 233)\end{array}$ \\
\hline $\begin{array}{l}\text { "Vorwendezeitraum } \\
\text { bzw. DDR-Zeit" } \\
\text { (1975 bis } 1989 / 90)\end{array}$ & 9 & $\begin{array}{c}283 \\
( \pm 90) \\
(119 \ldots 399)\end{array}$ & $\begin{array}{c}340 \\
( \pm 63) \\
(228 \ldots 432)\end{array}$ & $\begin{array}{c}46 \\
( \pm 8) \\
(31 \ldots 56)\end{array}$ & $\begin{array}{c}2153 \\
( \pm 424) \\
(1535 \ldots 2895)\end{array}$ & $\begin{array}{c}94 \\
( \pm 34) \\
(41 \ldots 145)\end{array}$ & $\begin{array}{c}321 \\
( \pm 74) \\
(216 \ldots 435)\end{array}$ \\
\hline Tertiärer Untergrund & 4 & $\begin{array}{c}16 \\
( \pm 5) \\
(11 \ldots 23) \\
\end{array}$ & $\begin{array}{c}38 \\
( \pm 9) \\
(31 \ldots 50) \\
\end{array}$ & $\begin{array}{c} \\
( \pm 1) \\
(6 \ldots 8) \\
\end{array}$ & $\begin{array}{c}110 \\
( \pm 36) \\
(76 \ldots 155) \\
\end{array}$ & $\begin{array}{c}4 \\
( \pm 2) \\
(2 \ldots 7) \\
\end{array}$ & $\begin{array}{c}27 \\
( \pm 7) \\
(21 \ldots 36) \\
\end{array}$ \\
\hline $\begin{array}{l}\text { Oberflächensedi- } \\
\text { ment (Gesamt- } \\
\text { fraktion; BoRN 1991) }\end{array}$ & 36 & $\begin{array}{c}225 \\
( \pm 60) \\
(103 \ldots 312) \\
\end{array}$ & $\begin{array}{c}327 \\
( \pm 44) \\
(174 \ldots 399) \\
\end{array}$ & n.b. & $\begin{array}{c}2490 \\
( \pm 430) \\
(1160 \ldots 3290) \\
\end{array}$ & $\begin{array}{c}78 \\
( \pm 19) \\
(29 \ldots 115) \\
\end{array}$ & $\begin{array}{c}286 \\
( \pm 64) \\
(146 \ldots 445) \\
\end{array}$ \\
\hline Zeitraum & $n$ & $\begin{array}{c}\mathrm{Cr} \\
\mathrm{mg} / \mathrm{kg}\end{array}$ & $\begin{array}{c}\mathbf{N i} \\
\mathrm{mg} / \mathrm{kg}\end{array}$ & $\begin{array}{c}\mathrm{Ba} \\
\mathrm{mg} / \mathrm{kg}\end{array}$ & $\underset{\mathrm{mg} / \mathrm{kg}}{\mathrm{U}}$ & $\begin{array}{l}\mathrm{P}_{2} \mathbf{O}_{5} \\
\mathrm{mg} / \mathrm{kg}\end{array}$ & $\begin{array}{c}\mathrm{S} \\
\mathrm{mg} / \mathrm{kg}\end{array}$ \\
\hline $\begin{array}{l}\text { August } 2002- \\
\text { Hochwasserlage }\end{array}$ & 2 & $\begin{array}{c}111 \\
( \pm 3) \\
(107 \ldots 111)\end{array}$ & $\begin{array}{c}76 \\
( \pm 5) \\
(69 \ldots 76)\end{array}$ & $\begin{array}{c}1112 \\
( \pm 124) \\
(1024 \ldots 120 \\
0) \\
\end{array}$ & $\begin{array}{c}19 \\
( \pm 2) \\
(17 \ldots 20)\end{array}$ & $\begin{array}{c}\mathbf{5 9 1 6} \\
( \pm 303) \\
(5701 \ldots 6130)\end{array}$ & $\begin{array}{c}\mathbf{2 2 7 6} \\
( \pm 334) \\
(2039 \ldots 2512)\end{array}$ \\
\hline $\begin{array}{l}\text { "Postwende- } \\
\text { zeitraum" (1989/90 } \\
\text { bis August 2002) }\end{array}$ & 13 & $\begin{array}{c}120 \\
( \pm 11) \\
(107 \ldots 151) \\
\end{array}$ & $\begin{array}{c}89 \\
( \pm 6) \\
(80 \ldots 102) \\
\end{array}$ & $\begin{array}{c}939 \\
( \pm 112) \\
(721 \ldots 1168) \\
\end{array}$ & $\begin{array}{c}30 \\
( \pm 9) \\
(20 \ldots 45) \\
\end{array}$ & $\begin{array}{c}10735 \\
( \pm 2878) \\
(7680 \ldots 17260) \\
\end{array}$ & $\begin{array}{c}\mathbf{5 9 5 1} \\
( \pm 2059) \\
(3901 \ldots 10950) \\
\end{array}$ \\
\hline $\begin{array}{l}\text { "Vorwendezeitraum } \\
\text { bzw. DDR-Zeit" } \\
\text { (1975 bis 1989/90) }\end{array}$ & 9 & $\begin{array}{c}216 \\
( \pm 38) \\
(158 \ldots 279)\end{array}$ & $\begin{array}{c}118 \\
( \pm 14) \\
(102 \ldots 137)\end{array}$ & $\begin{array}{c}1372 \\
( \pm 176) \\
952 \ldots 1564)\end{array}$ & $\begin{array}{c}74 \\
( \pm 22) \\
(43 \ldots 119)\end{array}$ & $\begin{array}{c}20931 \\
( \pm 8374) \\
(10280 \ldots 32370)\end{array}$ & $\begin{array}{c}11504 \\
( \pm 4872) \\
(4311 \ldots 18600)\end{array}$ \\
\hline Tertiärer Untergrund & 4 & $\begin{array}{c}83 \\
( \pm 7) \\
(77 \ldots 92) \\
\end{array}$ & $\begin{array}{c}32 \\
( \pm 2) \\
(29 \ldots 34) \\
\end{array}$ & $\begin{array}{c}522 \\
( \pm 58) \\
(465 \ldots 596) \\
\end{array}$ & $\begin{array}{c}52 \\
( \pm 55) \\
(3 \ldots 100) \\
\end{array}$ & $\begin{array}{c}2883 \\
( \pm 565) \\
(2400 \ldots 3670) \\
\end{array}$ & $\begin{array}{c}6382 \\
( \pm 2136) \\
(3179 \ldots 7544) \\
\end{array}$ \\
\hline $\begin{array}{l}\text { Oberflächensedi- } \\
\text { ment }{ }^{\text {a) }} \text { (Gesamt- } \\
\text { fraktion; BORN, 1996) }\end{array}$ & 36 & $\begin{array}{c}178 \\
( \pm 27) \\
(117 \ldots 247) \\
\end{array}$ & $\begin{array}{c}116 \\
( \pm 26) \\
(55 \ldots 186) \\
\end{array}$ & n.b. & n.b. & n.b. & n.b. \\
\hline & & & & & & & \\
\hline
\end{tabular}

Erläuterungen: Angegeben sind der Median (oben), die Standardabweichung (Mitte) und Minimumwert und Maximumwert (unten, in Klammer) der mittels RFA am Gesamtsediment $(<2 \mathrm{~mm})$ bestimmten Elementgehalte. Die Gehalte der Zeitabschnitte „Postwendezeit“ und „Vorwendezeit“ erfolgte auf der Basis der zeitlichen Zuordnung der Sedimentkernabschnitte nach den in der vorliegenden Arbeit beschriebenen Methoden. Eingegangen sind die Ergebnisse von insgesamt vier Kernen aus dem Friedersdorfer Becken und dem Hauptbecken des Bitterfelder Muldestausees. Den „tertiären Untergrund“ bilden die das Seesediment unterlagernden tertiären Schichten (ehemalige Tagebauoberfläche). a) nach BoRN (1996); n.b. ... nicht bestimmt. 
lometer unterhalb des Bitterfelder Muldestausees gelegenen Flusspegels Bad Düben; mittlerer Durchflusswert der Mulde seit 1975: ca. 64 $\mathrm{m}^{3} / \mathrm{s}$; Deutsches Gewässerkundliches Jahrbuch, LFU Sachsen-Anhalt) mit hoher Wahrscheinlichkeit zur Ausbildung makroskopisch deutlich sichtbarer Hochwasserlagen im Seesediment des Bitterfelder Muldestausees. In Abhängigkeit von der Depositionsposition im Stausee werden dabei Lagenmächtigkeiten im $\mathrm{cm}$-Bereich und darüber erreicht. Die Ereignislagen wechseln sich mit organikreichen Sedimentabschnitten (dunkle Lagen) ab, die verstärkte Phasen der Bioproduktion widerspiegeln, wobei die Bildung letzterer auf die saisonal wärmeren Abschnitte im Jahresgang (Früh-, Hoch- und Spätsommer) konzentriert sind.

Die genannte Konstellation führt dazu, dass mehrere, während einer Winterperiode stattgefundene Hochwässer, sich im Sediment mehrere aufeinanderfolgende und innerhalb einundderselben Winterperiode stattgefundene Hochwässer sich im Sediment nur in der Ausbildung einer makroskopisch sichtbaren Ereignislage widerspiegeln. Diese erscheint zwar entsprechend der Zahl der Hochwässer und ihres Verlaufes im Dünnschliffbild häufig gradiert, ist aber nicht von organikreichen, dunklen Lagen unterbrochen. Die Besonderheiten der winterlichen Hochwassersituationen werden im mikroskopischen, weniger im makroskopischen Bild des Lagenaufbaus der entsprechenden Ereignislage sichtbar. Hingegen führt jedes Sommerhochwasser zur Ausbildung einer eigenständigen makroskopisch sichtbaren Ereignislage im Seesediment. Dies allerdings nur, soweit der zeitliche Abstand zwischen aufeinanderfolgenden Sommerhochwässern für die Aktivierung der Bioproduktivität im See ausreicht. Die normale, biogen bestimmte Sommersedimentation, die zur Ausbildung einer dunklen Lage führt, wird bei Abwesenheit von Hochwässern in der darauffolgenden Winterperiode makroskopisch nicht unterbrochen, d.h. beim Auftreten von Wintern mit niedrigen bis mittleren Durchflüssen kommt es zu keiner Ausbildung einer makroskopisch sichtbaren, hellgefärbten und mächtigen klastischen Lage. Im mikroskopischen Bild erscheinen diese dunklen Lagen allerdings differenziert.

Bei der makroskopischen Zuordnung der Hochwasserereignisse zu den im Sediment des Kernes MUL1HW auftretenden Ereignislagen fanden die genannten, saisonal bedingten Effekte ihre Berücksichtigung (Tabelle 2).

Seit dem 1. Mai 1975, dem Beginn der Flutung des ehemaligen Tagebaurestloches Muldenstein durch die Mulde, wurden 33 Hochwässer mit einem mittleren Tagesdurchfluss größer 300 $\mathrm{m}^{3} / \mathrm{s}$ registriert (Flusspegel Bad Düben; Gewässerkundliches Jahrbuch, LFU Sachsen-Anhalt). Unter Berücksichtigung der oben erläuterten saisonal bedingten Effekte würden diese 33 Hochwässer (einschließlich des August-2002Hochwassers) sich in 22 makroskopisch sichtbaren und von dunklen organikreichen Lagen abgegrenzten Ereignislagen widerspiegeln, die insgesamt im seit 1975 zur Ablagerung gelangten Seesediment des Muldestausees sichtbar werden sollten (Tabelle 2).

Der hier vorgestellte Kern MUL1HW lässt einschließlich der während des August-2002Hochwassers abgesetzten Sedimentlage in summa 14 makroskopische Ereignislagen erkennen. Auf Grund der radiometrischen Ergebnisse $\left({ }^{137} \mathrm{Cs}\right)$ wird der Sedimentationszeitraum auf den Zeitraum vom Januar 1986 bis August 2002 eingegrenzt. In diesem Zeitraum wurden insgesamt 21 Hochwassersituationen (größer $300 \mathrm{~m}^{3} / \mathrm{s}$ ) registriert. Diese beinhalten 17 Winterhochwässer, verteilt auf 10 Winterperioden, und 4 Sommerhochwässer (Tabelle 2). Daraus ergibt sich, dass sich die registrierten 21 Hochwassersituationen entsprechend des oben erläuterten Konzeptes in 14 Ereignislagen im Sediment widerspiegeln müssten. Diese aus dem Modell abgeleitete Anzahl von Ereignislagen deckt sich mit den Feldbefunden, d.h. mit 
Tab. 2: Zeitliche Zuordnung von Ereignislagen in Sedimentkernen aus dem Bitterfelder Muldestausee zu Hochwasserereignissen der Mulde mit einem Tagesdurchflusswert $Q \geq 300 \mathrm{~m}^{3} / \mathrm{s}$ unter besonderer Berücksichtigung des Kernes MUL1HW (Daten Flusspegel Bad Düben, Angaben LfU Sachsen-Anhalt).

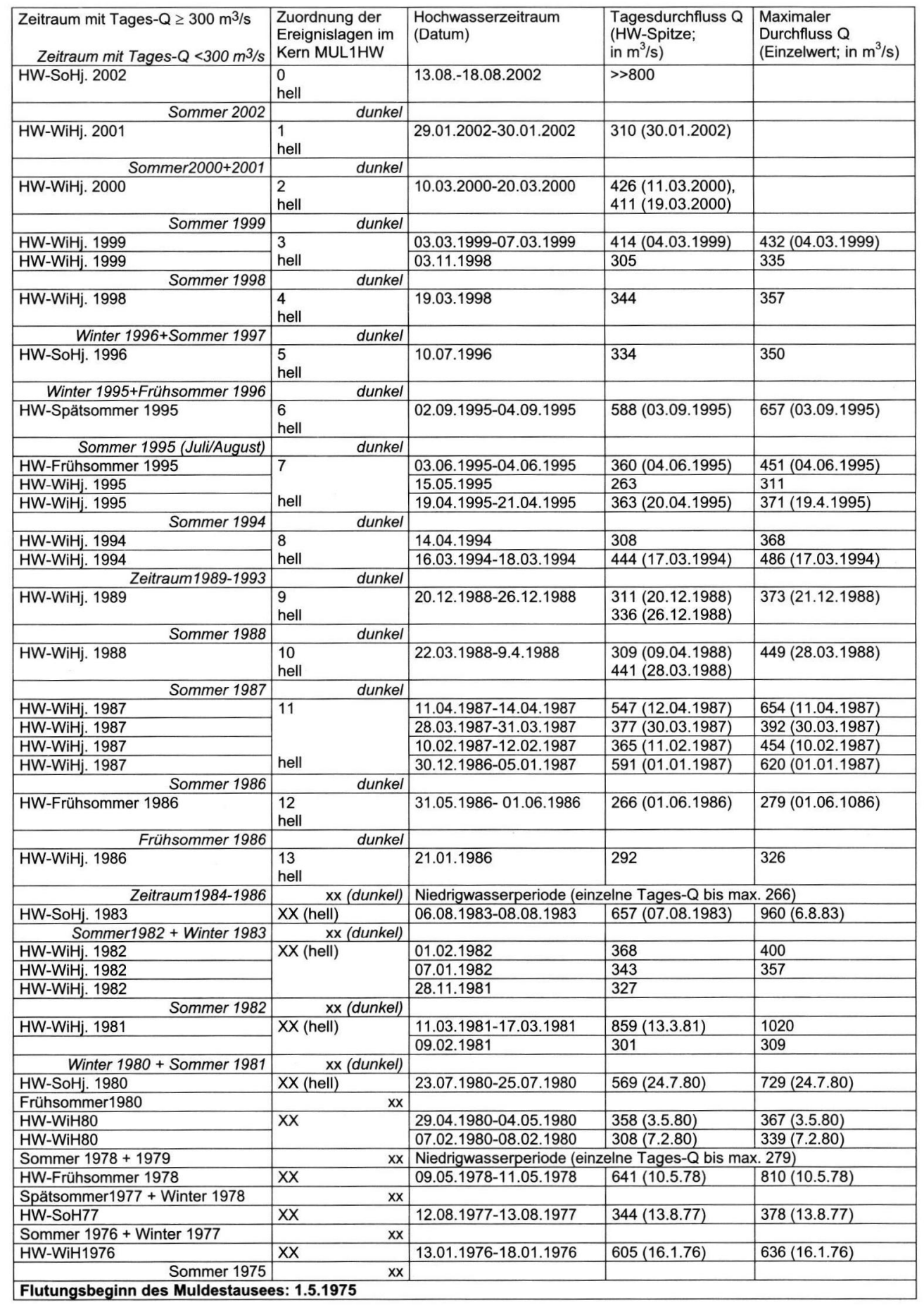


der im Kern MUL1HW gefundenen makroskopisch sichtbaren Anzahl von 14 Ereignislagen (Tabelle 2, Abb. 2).

Neben den radiometrischen Ergebnissen wird die Zuordnung der im Kern MUL1HW dokumentierten Ereignislagen zu den registrierten Hochwassersituationen der Jahre 1986 bis 2002 auch durch die elementgeochemischen Befunde gestützt. Der in Folge der "politischen Wende" 1989/90 begründete und im Seesediment des Bitterfelder Muldestausees nachgewiesene Rückgang der Gehalte an Schwermetallen (u.a. $\mathrm{Zn}, \mathrm{Cd}, \mathrm{Cu}, \mathrm{Ni}, \mathrm{Cr}$ ) und an anderen Indikatorelementen (z.B. Phosphor) fällt in den Bereich zwischen den registrierten Hochwasserereignissen der Winterperioden 1988 (Ereignislage Nr. 9) und 1994 (Ereignislage Nr. 10; Abb. 2, Tabelle 2). Er dokumentiert eine vierjährige Niedrigwasserperiode (1989-1993) und ist durch die Ausbildung einer dunklen organikreichen Lage ausgewiesen. Sie bildet den Abschluss des beschriebenen unteren ca. 0,14 m mächtigen Kernabschnittes, der auf Grund höherer Organikanteile makroskopisch einen deutlich dunkleren Eindruck macht und verschwommenere Lagengrenzen aufweist.

\section{Schlussfolgerungen}

Flussstauseen sind geeignete Sedimentfallen zur Rekonstruktion von Hochwässern und deren Auswirkungen in Vergangenheit und Gegenwart. Dabei erweist sich der Bitterfelder Muldestausee als Modellbeispiel, um den ereignisspezifischen realen Schwebstoffauftrag im Stausee bei Hochwassersituationen in Abhängigkeit verschiedener Einflussfaktoren (z.B. Durchfluss, Hochwasserverlauf u.a.) zu studieren. Insbesondere für die Hochwasserforschung historischer und geologischer Zeiten können die dabei gewonnenen Ergebnisse genutzt werden. Dies betrifft Schlussfolgerungen zur Sedimentationsrate, d.h. zum realen Auftrag an
Schwebstoff bzw. Feinstsediment in Seen, die einen Anschluss an ein Fließgewässer besitzen, und Schlussfolgerungen zur zeitlichen Fixierung von Ereignislagen in anderen geowissenschaftlich nutzbaren Hochwasserarchiven (z.B. Hochflutsedimente, Auelehm).

Die am Sedimentkern aus dem Bitterfelder Muldestausee erzielten Ergebnisse der zeitlichen Zuordnung von Ereignislagen zu spezifischen Hochwassersituationen lassen weiterhin praktische Schlussfolgerungen zur Hochwasservorsorge und zum Hochwassermanagement zu. Sie zeigen, dass trotz des hohen Wirkungsgrades der Sedimentfalle „Bitterfelder Muldestausee“ (weit über 90\% des gesamten von der Mulde transportierten Schwebstoffes gelangen im Flussstausee zur Ablagerung), der durch Hochwässer erfolgte reale Sedimentauftrag an Feinstschweb auf die Seebodenoberfläche pro Ereignis auch bei Extremhochwässern relativ gering ist und sich im cm-Bereich bewegt. Auf der Basis der historischen Häufigkeit von Hochwasserereignissen wird damit die Eignung des Bitterfelder Muldestausees als langjährig wirksame $\mathrm{Se}$ dimentfalle (mindestens 500 bis 700 Jahre) für das untere Mulde-Elbe-Gebiet bestätigt. Damit werden Möglichkeiten zur Einbindung anderer natürlich oder anthropogen geschaffener Staubereiche in das Hochwassermanagement von Flusseinzugsgebieten aufgezeigt.

\section{Danksagungen}

Die Untersuchungen wären ohne die freundschaftliche und uneigennützige Hilfe und die vielfältigen Erfahrungen unseres Freundes und Kollegen Professor Dr. B. Scharf (Magdeburg) nicht möglich gewesen. Sie reichten von der Logistik, über die Gewinnung der Kernproben, ihrer Lagerung bis hin zur sachgerechten Öffnung der Kerne. Für die zeitweilige Unterstützung bei den durchgeführten Bohrkampagnen möchten wir den Herren M. Herzog 
(Magdeburg), Dipl.-Geogr. St. Knetsch (Halle), Dr. M. Pirrung (Jena) danken. Für die Mitarbeit bei der Öffnung der im April 2002 entnommenen Sedimentkerne danken wir Dipl.-Ing. (FH) A. ArNold (Leipzig) recht herzlich. Die laborativen Arbeiten wurden in gewohnter sorgfältiger und gewissenhafter Art und Weise von Th. Berger (Leipzig; Glühverlust) und I. Fröhlich (Leipzig; RFA) durchgeführt. Dem Staatlichen Umweltfachamt LeIPZIG (Stufa) danken wir für die kostenlose Bereitstellung von Durchflussdaten der Mulde (Pegel Bad Düben), dem Wassersportclub Friedersdorf e.V. für die Möglichkeiten zur Unterstellung unserer Bootsausrüstungen. Dipl.-Min. W. Czegka, M.Sc. und Dipl.Chem. Chr. Hanisch (Leipzig) möchten wir für zahlreiche fachliche Hinweise zur Probenchemie und -analytik danken. Teilergebnisse der Arbeiten erfuhren 2003/04 eine finanzielle Förderung im Rahmen des BMBF-AdhocHochwasserprojektes (Nr. 0330492).

\section{Literatur}

Beuge, P., Greif, A., Hoppe, T., Klemm, W., Kleebberg, R., Kluge, A., Mosler, U., Starke, R., Alfaro, J., Haurand, M., Knöchel, A. \& Meyer, A. (1994): Erfassung und Beurteilung der Schadstoffbelastung des Muldesystems. - In: Statusberichte 1994 "Die Belastung der Elbe Teil I- Elbenebenflüsse" Bundesministerium für Bildung, Wissenschaft, Forschung und Technologie: 27-37; Forschungszentrum Karlsruhe $\mathrm{GmbH}$.

Böhm M. \& Pfeffer K.H. (1990): Geoökologische Studien in der durch den Reaktorunfall von Tschernobyl hoch kontaminierten Region südlich Tannheim/Oberschwaben.Tübinger Geowissenschaftliche Arbeiten, Reihe C, 7: 31-65; Universität Tübingen.

Born J. (1996): Sedimentgeochemie des
Muldestausees bei Bitterfeld. - Heidelberger Beitr. Umwelt-Geochemie, 9: 226 S.; Ruprecht-Karls-Universität Heidelberg.

Einsele G. \& Pfeffer K.-H. (1990): Untersuchungen über die Auswirkungen des Reaktorunfalls von Tschernobyl auf Böden, Klärschlamm und Sickerwasser im Raum von Oberschwaben und Tübingen.Tübinger Geowissenschaftliche Arbeiten, Reihe C, 7: 151 S.; Universität Tübingen.

JeNDRYsCHIK K. (2003): Schwebstofffrachten der Mulde am Zufluss zum Bitterfelder Muldestausee in den Jahren von 1991 bis 2001 und die Sedimentation im See. - Jb. Sächs. Akad. d. Wiss. zu Leipzig, 20012002: 240-249; Stuttgart/Leipzig.

Junge, F.W., Duckheim, W., Morgenstern \& P., Magnus, M. (2001): Sedimentologie und Geochemie obereozän-unteroligozäner Typusprofile aus dem Weißelsterbecken (Tagebau Espenhain). - Mauritiana 18 (1): 25-59; Altenburg.

Junge, F.W., Hanisch, Chr., Zerling, L., Czegka, W., Arnold, A., Jendryschik, K.; Morgenstern, P., Treutler, H.C. \& Scharf, B. (2004): Arbeitspaket 3.6.: Untersuchungen im Bitterfelder Muldestausee zu den Auswirkungen des Hochwassers vom August2002 auf die Schwermetallgehalte von Schwebstoffen und Sedimenten.- In: W. Geller, K. Ockenfeld, M. Böhme, A. Knöchel (Hrsg.): Schadstoffbelastung nach dem Elbe-Hochwasser 2002 UFZ Leipzig-Halle $\mathrm{GmbH}$ u.a., Endbericht des BMBF-Adhoc-Verbundprojektes PTJ0330492: 136158; Leipzig.

Landesamt für Umweltschutz SachsenAnhalt. Jährlich. Deutsches Gewässerkundliches Jahrbuch. Elbegebiet. Teil 1.

Magnus, M. (1998): Mikroskopische Phasenund Kornformanalyse klastischer Sedimentite mittels Bildanalyse am beispiel des Unterturons der Elbezone. - Diss., TU Ber- 
gakademie Freiberg, Institut für Geologie: 118 S.; Freiberg.

Zerling, L., Müller A., Jendryschik, K., Hanisch, C. \& Arnold, A. (2001): Der Bitterfelder Muldestausee als Schadstoff- senke. Entwicklung der Schwermetallbelastung von 1992 bis 1997. - Abh. d. Sächs. Akad. d. Wiss. zu Leipzig, Math.-nat. Kl., 59, (4): 69 S.; Stuttgart (Hirzel). 